\title{
LARVICULTURA DE SÁBALO (Prochilodus lineatus) CON DIFERENTES TIEMPOS EN LABORATORIO Y ESTANQUE
}

\author{
Comolli, Javier A.*; González, Alfredo O.; Agüero, Carlos H.; Sánchez, Sebastián; Roux, Juan P. \\ Instituto de Ictiología del Nordeste - Facultad de Ciencias Veterinarias - UNNE. \\ Sargento Cabral 2139, (3400) Corrientes. Tel.: (0379) 4425753. Int. 177. \\ * e-mail: javiercomolli@gmail.com
}

\section{RESÚMEN}

El objetivo del presente trabajo fue evaluar la supervivencia (TS), peso medio(PM) y biomasa (BM) producida en larvicultura de sábalo (Prochilodus lineatus) con diferentes tiempos de permanencia en laboratorio y estanques. La experiencia se llevó a cabo en las instalaciones del Instituto de Ictiología del Nordeste (INICNE). Tuvo una duración de 35 días. Las larvas utilizadas tenían tres días de eclosión. El ensayo conto con cinco tratamientos: T1 (estanque durante 35 días); T2 ( 7 días en laboratorio y 28 días en estanque); T3 (14 días en laboratorio y 21 días en estanque); T4 (21 días de laboratorio y 14 días de estanque); T5 (21 días en laboratorio y 14 días en estanque, con diferentes tipos de alimento en la etapa de laboratorio). En la etapa de laboratorio a los peces se les suministró alimento balanceado con $28 \%$ de proteína bruta, excepto el T5 que fue alimentado con nauplios de Artemia salina. En estanque la dieta consistió en el mismo alimento balanceado más la producción primaria del estanque. Para el laboratorio se acondicionaron acuarios con 5 litros de agua, con renovación y aireación continua. La densidad de peces fue de 100 larvas por litro. En estanque se acondicionaron jaulas con una densidad 90ejemplares por jaula. Cada tratamiento tuvo tres replicas. Se procedió al recuento y pesaje de la totalidad de los peces, datos que se emplearon para estimar la TS, PM y BM. De los 5 tratamientos el T1, T2 y T3 no tuvieron diferencias significativas $(p \leq 0,05)$ para las tres variables estudiadas. En cambio, T5 tuvo una TS $(98,52 \%)$ que se diferenció $(p \geq 0,05)$ de T4 $(53,33 \%)$. T3 se diferenció significativamente en PM $(1,02 \mathrm{~g})$ de T5 $(0,30 \mathrm{~g})$ y en Biomasa $(69,55 \mathrm{~g})$ de T4 $(32,79 \mathrm{~g})$ y T5 $(26,61 \mathrm{~g})$. La combinación de larvicultura en laboratorio hasta 14 días y luego en estanques en cuanto a TS, PM y Biomasa es aceptable a nivel productivo. Se apreció una elevada heterogeneidad de tamaños en los tratamientos con alimento balanceado, no así en el T5. La alimentación con $A$. salina hasta 21 días mostró una alta TS, pero muy bajos PM y Biomasa.

Palabras Clave: Prochilodus lineatus, alimentación, supervivencia, peso, biomasa.

\begin{abstract}
The aim of this study was to evaluate survival, average weight and biomass produced in larviculture of Prochilodus lineatus with different residence times in laboratory ponds. The experience was carried out at the facilities of the Northeast Ichthyology Institute (INICNE). It lasted 35 days. The larvae used had three days of hatching. The test consisted of five treatments (T): T1 pond for 35 days; T2 7 days at laboratory and 28 days in the pond; T3 14 days at laboratory and 21 days in the pond; T4 21 days of laboratory and 14 days of pond; T5 21 days at the laboratory and 14 days in the pond, with different type of food at laboratory stage. At laboratory fish were fed with $28 \%$ crude protein, except T5 which was fed with Artemia salina nauplii. Pond feeding consist of balanced meal plus the primary pond production. Aquarium laboratory were filled with $5 \mathrm{lt}$ of water, and provided with continuous aeration system. The density of fish was 100 per It. In the pond, cages with a density of 90 fish per cage were conditioned. Each treatment had three replicates. We proceeded to the counting and weighing of all the fish, data that were used to estimate the survival rate (TS), the average weight (MP) and Biomass Of the 5 treatments, $\mathrm{T} 1, \mathrm{~T} 2$ and $\mathrm{T} 3 \mathrm{did}$ not have significant differences $(\mathrm{p} \leq 0.05)$ for the three variables studied. On the other hand, T5 had TS (98.52\%) that differed ( $\mathrm{p} \geq 0.05)$ from T4 (53.33\%). T3 differed significantly in PM (1.02g) from T5 $(0.30 \mathrm{~g})$ and in Biomass $(69.55 \mathrm{~g})$ from T4 $(32.79 \mathrm{~g})$ and T5 (26.61g). The combination of larviculture at the laboratory up to 14 days and then in ponds in terms of TS, PM and biomass is acceptable at a productive level. A high heterogeneity of sizes was observed in treatments with balanced feed, but not in T5. The feeding with $A$. salina until 21 days showed a high TS but very low PM and Biomass.
\end{abstract}

Key Words: Prochilodus lineatus, larviculture, feeding, weight, biomasas.

Recibido: 20/may/2019. Aceptado: 07/ago/2019 


\section{INTRODUCCIÓN}

El sábalo (Prochilodus lineatus) se distribuye en Argentina por las cuencas de los ríos Paraná, Paraguay, Uruguay, Río de la Plata, Bermejo y Pilcomayo (Ringuelet et al., 1967). De hábitos alimenticios iliófagos, está adaptado para consumir detritos orgánicos del fondo de ríos y lagunas (Atencio, 2001; Ringuelet et al., 1967; Sverlij et al., 1993), siendo la especie de mayor importancia pesquera comercial de agua continental de la Argentina (Flores Nava y Münzemayer, 2010). Estas características lo convierten en una buena opción para la producción en policultivo con otras especies de peces (Campos, 2010; Graeff y Tomaselli, 2011), lo que permitiría incrementar la biomasa de un estanque sin aumentar la oferta de alimento balanceado.

$P$. lineatus, podría incorporarse en cultivos como acompañante del pacú (Piaractus mesopotamicus), bajo sistema semintensivo en estanques de tierra excavados (Hepher y Pruginin, 1985). Como en toda piscicultura la etapa de larvicultura es de gran importancia. Constituye el período de mayor mortalidad, siendo el lapso donde los peces presentan más rápido crecimiento y desarrollo. Esto la convierte en una etapa crítica del ciclo productivo, donde se debe garantizar la producción de una cantidad de juveniles suficiente como para abastecer a los piscicultores de la región.

Larvas de diferentes especies de peces son criadas a densidades variables bajo condiciones controladas de laboratorio, que van desde de 10 a 300 larvas por litro, así como en estanques a cielo abierto, donde las densidades van de 100 a 500 larvas metro cubico (Atencio et al., 2003). Si bien cada sistema tiene ventajas y desventajas, la larvicultura intensiva se generalizó ya que permite mejorar la tasa de supervivencia y si bien suele presentar reducción del crecimiento, estrés y aparición de enfermedades (Barcellos et al., 2000; Jobling 1994; King et al., 2007), posibilita contar con un elevado número de individuos al final de esta etapa.

Un factor que influye en la respuesta de las larvas de peces a la densidad de cría es la alimentación (Dou et al., 2003), resultando determinante el uso de alimento vivo, el que asegura altas tasas de supervivencia (Kolvoski, 2001) a pesar del inconveniente de ser costoso y no cubrir la totalidad de los requerimientos a medida que las larvas crecen (Hernández et al., 2006; Luchini y Avendaño, 1985).

El objetivo del presente trabajo fue evaluar la TS, PM y BM producidos en larvi- cultura de sábalo con diferentes tiempos de permanencia en laboratorio y estanques.

\section{MATERIALES Y MÉTODOS}

La experiencia se llevó a cabo en las instalaciones del Instituto de Ictiología del Nordeste (INICNE) (Corrientes, Argentina), con una duración de 35 días. Se utilizaron reproductores propios y la inducción hormonal para el desove y espermiación se realizó con extracto de hipófisis de ejemplares de la misma especie. Las larvas utilizadas tenían tres días de eclosión, con un Peso Medio (PM) de 43,2 $\pm 8,4 \mathrm{mg}$ y habían empezado a alimentarse.

El ensayo contó con cinco tratamientos: T1 (en estanque durante 35 días); T2 (7 días en laboratorio y 28 días en estanque); T3 (14 días en laboratorio y 21 días en estanque); T4 (21 días de laboratorio y 14 días de estanque); T5 (21 días laboratorio y 14 días en estanque con diferente alimentación en laboratorio).

En el laboratorio, los alevinos fueron alimentados con balanceado con $28 \%$ de Proteína Bruta (PB), excepto el T5 que fue alimentado con nauplios de $A r$ temia salina, comenzando con 100 nauplios por individuo durante los primeros 7 días del ensayo y luego 150 nauplios hasta pasar al estanque. En estanques, la alimentación fue con alimento balanceado, con $28 \%$ de $\mathrm{PB}$, más la producción primaria del estanque.

Para el laboratorio se acondicionaron acuarios con 5 litros de agua, con renovación y aireación continua. La densidad de peces fue de 100 por litro (Roux et al., 2015). En estanques, se acondicionaron jaulas con una densidad de 90 peces por jaula, alcanzando una densidad de 360 ejemplares por metro cúbico (Fig.1).

Diariamente a las 9 y 16 horas se registraron parámetros de calidad del agua: $\mathrm{O}_{2}$ disuelto en $\%$ y mg por litro, temperatura del agua y temperatura ambiente

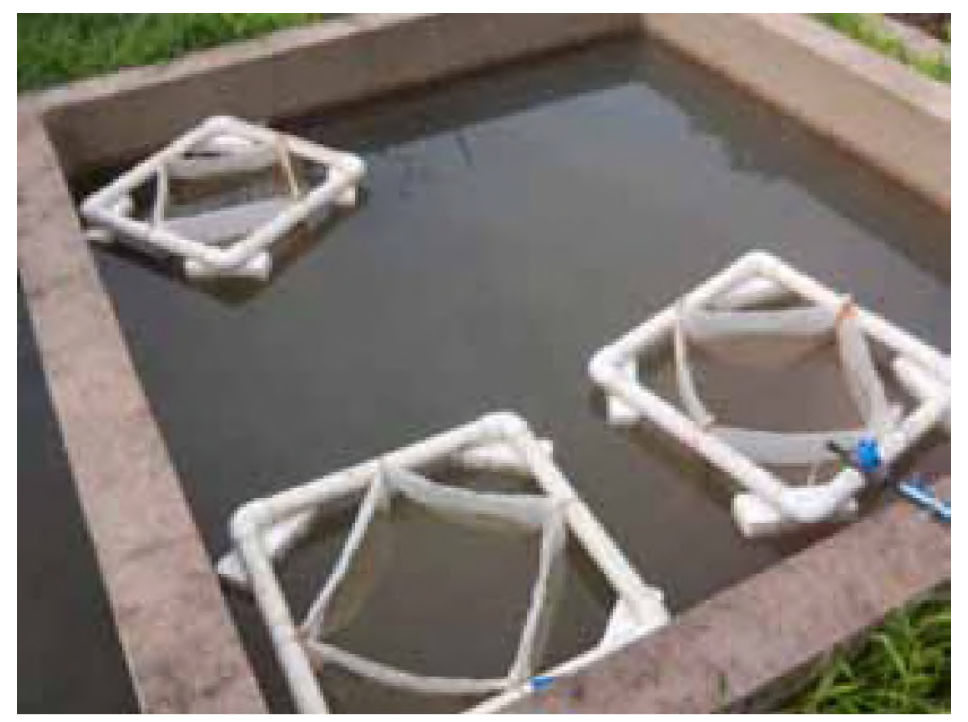

Figura 1. Estanque de larvicultura semintensiva. 
Tabla 1. Promedio de los valores de Calidad de agua en acuarios para larvicultura de sábalo (P. lineatus)

\begin{tabular}{|c|c|c|c|c|c|c|c|c|}
\hline \multirow[b]{2}{*}{ Trat. } & \multicolumn{3}{|c|}{$9 \mathrm{hs}$} & \multicolumn{3}{|c|}{$16 \mathrm{hs}$} & \multirow[b]{2}{*}{$\mathrm{pH}$} & \multirow{2}{*}{$\begin{array}{l}\text { Cond. } \\
(\mu \mathrm{S} / \mathrm{cm})\end{array}$} \\
\hline & $\begin{array}{l}\text { Temperatura } \\
\text { Agua }(\stackrel{\circ}{ } \mathrm{C})\end{array}$ & $\begin{array}{l}\mathrm{O} 2 \\
(\%)\end{array}$ & $\begin{array}{c}\mathrm{O} 2 \\
(\mathrm{mg} / \mathrm{l})\end{array}$ & $\begin{array}{c}\text { Temperatura } \\
\text { Agua }\left({ }^{\circ} \mathrm{C}\right)\end{array}$ & $\begin{array}{l}\mathrm{O} 2 \\
(\%)\end{array}$ & $\begin{array}{c}\mathrm{O} 2 \\
(\mathrm{mg} / \mathrm{l})\end{array}$ & & \\
\hline 2 & 25,8 & 75,6 & 6,2 & 25,4 & 77,5 & 6,3 & 6,3 & 89,2 \\
\hline 3 & 25,7 & 74,3 & 6,3 & 25,7 & 76,1 & 6,3 & 6,3 & 89,0 \\
\hline 4 & 25,8 & 70,9 & 6,4 & 25,8 & 73,6 & 6,0 & 6,3 & 89,7 \\
\hline 5 & 26,1 & 71,4 & 6,1 & 26,0 & 54,8 & 4,5 & 6,3 & 92,0 \\
\hline
\end{tabular}

Trat:: Tratamiento; $\mathrm{O}_{2}(\mathrm{mg} / \mathrm{lt})$ : Concentración de Oxígeno; $\mathrm{O}_{2}(\%)$ : Saturación de Oxígeno; Cond.: Conductividad del agua

Tabla 2. Promedio de los valores de calidad de agua en estanques de cemento para larvicultura de Sábalo $(P$. lineatus)

\begin{tabular}{|c|c|c|c|c|c|c|c|c|c|}
\hline \multirow[b]{2}{*}{ Trat. } & \multicolumn{3}{|c|}{$9 \mathrm{hs}$} & \multicolumn{3}{|c|}{$16 \mathrm{hs}$} & \multirow[b]{2}{*}{$\mathrm{pH}$} & \multirow{2}{*}{$\begin{array}{l}\text { Cond. } \\
(\mu \mathrm{S} / \mathrm{cm})\end{array}$} & \multirow{2}{*}{$\begin{array}{r}\text { Secch } \\
(\mathrm{cm})\end{array}$} \\
\hline & $\begin{array}{l}\text { Temperatura } \\
\text { Agua (ํ) }\end{array}$ & $\begin{array}{l}\mathrm{O} 2 \\
(\%) \\
\end{array}$ & $\begin{array}{c}\mathrm{O} 2 \\
(\mathrm{mg} / \mathrm{l}) \\
\end{array}$ & $\begin{array}{l}\text { Temperatura } \\
\text { Agua }\left({ }^{\circ} \mathrm{C}\right) \\
\end{array}$ & $\begin{array}{l}\mathrm{O} 2 \\
(\%) \\
\end{array}$ & $\begin{array}{c}\mathrm{O} 2 \\
(\mathrm{mg} / \mathrm{l}) \\
\end{array}$ & & & \\
\hline 1 & 27,3 & 86,8 & 7,0 & 28,5 & 95,8 & 8,0 & 7,0 & 112,5 & 37 \\
\hline 2 & 27,1 & 81,0 & 6,9 & 28,2 & 89,4 & 7,0 & 6,9 & 115,6 & 42 \\
\hline 3 & 27,2 & 79,5 & 6,8 & 28,5 & 93,9 & 7,6 & 6,9 & 111,5 & 39 \\
\hline 4 & 27.4 & 85,3 & 6,9 & 28,4 & 92,8 & 7,7 & 6,9 & 112,8 & 45 \\
\hline 5 & 27.1 & 83,0 & 7,0 & 28,6 & 93,1 & 7,9 & 7,1 & 115,1 & 43 \\
\hline
\end{tabular}

Trat:: Tratamiento; $\mathrm{O}_{2}(\mathrm{mg} / \mathrm{lt})$ : Concentración de Oxígeno; $\mathrm{O}_{2}(\%)$ : Saturación de Oxígeno; Cond.: Conductividad del agua

máxima y mínima en ${ }^{\circ} \mathrm{C}$. Dos veces por semana se registraron valores de conductividad, $\mathrm{pH}$ y transparencia de estanque por medio del disco de Secchi.

Cada tratamiento tuvo tres réplicas. Al final de la experiencia se procedió al recuento y pesaje de la totalidad de los peces, datos que se emplearon para estimar Tasa de Supervivencia (TS), PM y BM. Los resultados obtenidos se evaluaron mediante ANOVA a una vía para cada una de las variables analizadas (Di Rienzo et al, 2012).

\section{RESULTADOS Y DISCUSIÓN}

Las variables estudiadas de calidad de agua se encontraron dentro del rango adecuado para la cría de la especie (Tabla 1 y Tabla 2).

Los valores de las condiciones adecuadas de $\mathrm{pH}$ para peces de agua dulce varían entre 6 y 9 (Boyd, 1998). En un trabajo con alevines de Rhamdia quelen se comprobó que variaciones entre 4 y 9,5 no afectan la TS tras 4 días de exposición (Townsend y Baldisserotto, 2001; Zaions y Baldisserotto, 2000). En ambientes naturales los alevines de $P$. lineatus se desarrollan normalmente con $\mathrm{pH}$ que varía entre 5,1 y 9,1 (Thomaz et al., 1997). En producción el sábalo tolera variaciones de 4,08 a 9,72 sin presentar mortalidad, al menos por 5 días (Zaniboni-Filho et al., 2002). Los valores del ensayo se encuentran dentro de los rangos para la cría de esta especie.

De los 5 tratamientos el T1, T2 y T3 no tuvieron diferencias significativas $(p \leq 0,05)$ para las tres variables estudiadas. En cambio, T5 tuvo una TS $(98,52 \%)$ que se diferenció $(p \geq 0,05)$ de T4 (53,33\%). A su vez, $\mathrm{T} 3$ con $\mathrm{PM}=1,02 \mathrm{~g}$ y Biomasa $=69,55 \mathrm{~g}$, se diferenció significativamente en PM de T5 $(0,30 \mathrm{~g})$ y en Biomasa de T4 $(32,79 \mathrm{~g})$ y T5 $(26,6 \mathrm{gg})$ (Tabla 3$)$.

En Prochilodus scrofa, Korbestein y Durigan (2001) obtuvieron valores en esta etapa de crecimiento de $1,67 \mathrm{~g}$ de PM y $71,6 \%$ de TS tomados a los 68 días de vida, en este caso la densidad era de 1 larva por litro y se usaron alimento balanceado en todo el ensayo con un $35 \%$ de $\mathrm{PB}$. El mayor PM en relación al trabajo informado se podría atribuir a la duración del mismo ya que fue casi del doble. La TS es similar a las obtenidas en los T1 y T3, e inferior a los T2 y T5.

Observando valores de PM en poslarvas de Prochilodus argenteus criadas en sistemas intensivos, con diferentes niveles de PB y durante 25 días, pode-

Tabla 3. Valores medios de los parámetros productivos en alevinos de sábalo ( $P$. lineatus) criados en diferentes tiempos en sistemas intensivos y semiintensivos.

\begin{tabular}{|c|c|c|c|}
\hline & $\mathrm{TS}(\%)$ & $\mathrm{PM}(\mathrm{g})$ & Biomasa $(\mathrm{g})$ \\
\hline T 1 & $73,33_{\mathrm{AB}}$ & $0,85_{\mathrm{B}}$ & $53,52_{\mathrm{ABC}}$ \\
\hline T 2 & $88,15_{\mathrm{AB}}$ & $0,73_{\mathrm{AB}}$ & $55,61_{\mathrm{BC}}$ \\
\hline T 3 & $76,67_{\mathrm{AB}}$ & $1,02_{\mathrm{B}}$ & $69,55_{\mathrm{C}}$ \\
\hline T 4 & $53,33_{\mathrm{A}}$ & $0,68_{\mathrm{AB}}$ & $32,79_{\mathrm{AB}}$ \\
\hline T 5 & $98,52_{\mathrm{B}}$ & $0,30_{\mathrm{A}}$ & $26,61_{\mathrm{A}}$ \\
\hline
\end{tabular}

TS: Tasa de Supervivencia; PM: Peso Medio. Letras diferentes representan diferencias significativas entre tratamientos $(p<0,05)$. 
mos apreciar valores de $0,20 \mathrm{~g}$ con niveles de $36 \%$ de PB y 0,13 g para $32 \%$ de $P B$. Valores todos inferiores a los presentes con 35 días. En el caso de la TS el mejor resultado fue de $67,35 \%$ en el tratamiento con $36 \%$ de PB lo que es inferior a los esperados para sistemas intensivos y reflejados en este trabajo tanto para intensivos y semintensivos (Santos, 2013).

En $P$. lineatus en sistemas intensivos de larvicultura, durante 28 días se evaluaron la combinación de Plancton y alimento balanceado, con variaciones de 24 a $30 \%$ de PB, presentaron diferencias significativas entre el PM y TS de los tratamientos donde solo se dieron plancton y $\mathrm{AB}$ de aquellos que combinaron plancton y $A B$, indistintamente si fuera con $24 \%$ o $30 \%$ de $\mathrm{PB}$, siendo la combinación de alimentos más eficiente desde el punto de vista del PM y TS $(2,26$ mg y 98,75\%) (Furuya et al., 1999). Estos valores son comparables en relación a la TS del T5 informado, pero muy inferiores a los PM de todos los tratamientos del ensayo. Esto se debe a que la larvicultura intensiva se caracteriza por altas TS y bajas ganancias de peso (Luchini y Avendaño, 1985).

En larvicultura intensiva de Prochilodus magdalenae como primera alimentación se determinó que el nauplio de Artemia sp. es el mejor alimento para esta etapa por ganancia de peso y supervivencia y propone como mínimo tres días de este tipo de alimentación antes de pasar los peces a estanque. Los valores obtenidos son PM 0,01 g y $86,3 \%$ de TS tomados a los siete días del comienzo de la alimentación (Atencio et al., 2003). En este caso, si bien no se trata de la misma especie, si lo son la densidad (50 larvas por litro) y el sistema de trabajo del ensayo. Los valores comparables son con los del T5, pero debe tenerse presente que este se extendió por 28 días y las densidades de este tratamiento fueron de 100 larvas por litro.

\section{CONCLUSIÓN}

De los resultados se aprecia que para $P$. lineatus la combinación de larvicultura en laboratorio hasta 14 días y estanque hasta completar el período de cría permite obtener buenos resultados a nivel productivo en cuanto a los promedios de TS, PM y Biomasa al final de la cría.

La alimentación con $A$. salina hasta 21 días determina bajos PM y Biomasa, pero al tener una alta TS se podría aprovechar el crecimiento compensatorio de esta especie en etapas posteriores y de esta forma tener mayor cantidad de juveniles para engorde.

\section{BIBLIOGRAFÍA}

Atencio, V. (2001). Producción de alevinos de especies nativas. Rev MVZ Córdoba. 6:(1), 9-14.
Atencio, V., Kerguelén, E., Wadnipar, L., Narváez, A (2003). Manejo de la primera alimentación del bocachico (Prochilodus magdalenae). Rev MVZ Córdoba. 8:(1), 254-260.

Barcellos, L.J.G., Souza, S.D., Woehl, V.M. (2000). Estresse em peixes: fisiologia da resposta ao estresse, causas e conseqüências (Revisão). Bol Inst Pesca, 26 (1): 99-111.

Boyd, C.E. (1998). Water quality management for pond aquaculture: research and development. International Center for Aquaculture and Aquatic Environments. 43: $1-37$.

Campos, J. (2010). Género Prochilodus (sábalo). En: Peces nativos de agua dulce de América del Sur de interés para la acuicultura: Una síntesis del estado de desarrollo tecnológico de su cultivo (Flores Nava, A.; Brown, A.; Ed.) Serie Acuicultura en Latinoamérica, Número 1. FAO, 200 pp.

Koberstein, T.C.R.D., Durigan, J.G. (2001). Produção de larvas de curimbatá (Prochilodus scrofa) submetidas a diferentes densidades de estocagem e niveis de proteína bruta nas dietas. Ciência Rural, 31(1): 123-127. https:// dx.doi.org/10.1590/S0103-84782001000100020

Di Rienzo, J.A., Casanoves, F., Balzarini, M.G., Gonzalez, L., Tablada, M., Robledo, C.W. (2012) InfoStat versión 2012. Grupo InfoStat, FCA, Universidad Nacional de Córdoba, Argentina. http://www.infostat.com.ar

Dou, S.; Masuda, R.; Tanaka, M.; Tsukamoto, K. (2003). Identification of factors affecting the growth and survival of the settling Japanese flounder larvae, Paralichthys olivaceus. Aquaculture. 218(1), 309-327. https://doi. org/10.1016/S0044-8486(02)00531-8

Furuya, V.R.B., Hayashi, C., Furuya, W.M., Soares, C.M., Galdioli, E.M. (1999). Influência de plâncton, dieta artificial e suacombinação, sobre o crescimento e sobrevivência de larvas de curimbatá (Prochilodus lineatus). Acta Scientiarum. Animal Sciences, 21 (3), 699703.

Flores Nava, A., Münzemayer, C. (2010). Diagnóstico de oportunidades de transferencia de tecnología en acuicultura y pesca continental en países de América del Sur. Serie Acuicultura en Latinoamérica, Número 2. FAO, 67 pp.

Graeff, A., Tomaselli, A. (2011). Policultivo de carpas com introdução crescente do Curimatã (Prochilodus scropha) como espécie principal. REDVET-Rev. electrón. vet. 12(10).

Hernández, D., Domitrovic, H., Sánchez, S. (2006). Evaluación de diferentes dietas en la primera alimentación del Bagre sudamericano (Rhomdia quelen). IV Congreso Iberoamericano Virtual de Acuicultura, CIVA: 11511155.

Hepher, B., Pruginin, Y. (1985). Cultivo de peces comerciales. Editorial Limusa, 316p, México.

Jobling, M. (1994). Fish bioenergetics, Chapman and Hall, London, $294 \mathrm{p}$. 
King, N.J., Howell, W.H., Huber, M., Bengtson, D.A. (2000). Effects of larval stocking density on laboratory-scale and commercial-scale production of summer flounder Paralichthys dentatus. Journal of the World Aquaculture Society, 31(3): 436-445. https://doi. org/10.1111/j.1749-7345.2000.tb00893.x.

Kolkovski, S. (2001). Digestive enzymes in fish larvae and juveniles-implications and applications to formulated diets. Aquaculture 200: 181-201. https://doi. org/10.1016/S0044-8486(01)00700-1

Luchini, L., Avendaño, T. (1985). Primer alevinaje de bagre sudamericano, Rhamdia sapo (Val.) Eig. en condiciones controladas. Revista de la Asociación de Ciencias Naturales del Litoral. 16(2):137-147

Ringuelet, R., Aramburu, R., Alonso de Aramburu, A. (1967). Los peces argentinos de agua dulce. Comisión de investigación científica. La Plata, Argentina. 602 pp.

Roux, J.P., González, A.O., Ortiz, J., Sánchez, S., ComoIli, J. (2015). Larvicultura intensiva de sábalo (Prochiloduslineatus) con diferentes densidades de cría. Rev. vet 26 (2): 143-146.

Santos, A.E. (2013). Larvicultura de Prochilodus argenteus (curimatã) com diferentes dietas comerciais e frequências alimentares. Dissertação (Mestradoem Zootecnia) - Faculdade de Ciências Agrárias Universidade Federal dos Vales do Jequitinhonha e Mucuri. Diamantina: UFVJM. $72 \mathrm{p}$.
Sverlij, S., Espinach Ros, A., Ortiz, G. (1993). Sinopsis de los Datos Biológicos y Pesqueros del Sábalo Prochilodus lineatus (Valenciennes, 1847). FAO. Sinopsis sobre la pesca $N^{\circ} 154.64$ pp. http://www.fao.org/3/t0808s/ t0808s.pdf

Thomaz, S.M., Roberto, M.D., Bini, L.M., Vazzoler, A.E., Agostinho, A.A. (1997). Caracterização limnológica dos ambientes aquáticos e influência dos níveis fluviométricos. Vazzoler A E, Agostinho AA, Hahn N S (Ed.). A planície de inundação do alto rio Paraná: aspectos físicos, biológicos e socioeconômicos. Eduem: 73-102.

Townsend, C.R., Baldisserotto, B. (2001). Survival of silver catfish fingerlings exposed to acute changes of water $\mathrm{pH}$ and hardness. Aquaculture International 9: 413-419. https://doi.org/10.1023/A:1020592226860

Zaions, M.I., Baldisserotto B. (2000). $\mathrm{Na}^{+}$and $\mathrm{K}^{+}$body levels and survival of fingerlings of Rhamdia quelen (siluriformes, pimelodidae) exposed to acute changes of water pH. Ciência Rural, 30(6), 1041-1045. https:// dx.doi.org/10.1590/S0103-84782000000600020

Zaniboni-Filho, E., Meurer, S, Golombieski J.I., Silva, L., Baldisserotto, B. (2002). Survival of Prochilodus lineatus (Valenciennes) fingerlings exposed to acute $\mathrm{pH}$ changes. Acta Scientiarum. 24: 917-920. 